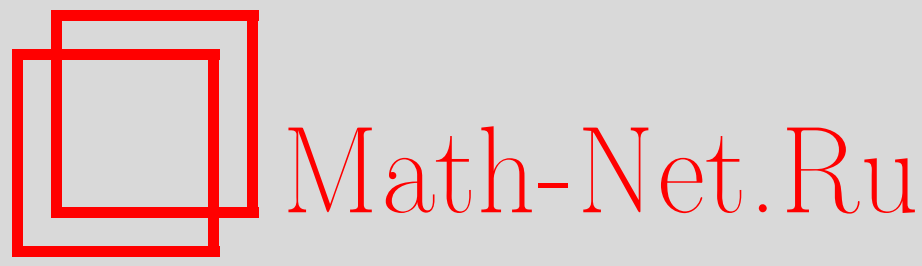

В. Р. Фаталов, Некоторые асимптотические формулы для гауссовской меры Боголюбова, ТМФ, 2008, том 157, номер 2, 286-308

DOI: https://doi.org/10.4213/tmf6280

Использование Общероссийского математического портала Math-Net.Ru подразумевает, что вы прочитали и согласны с пользовательским соглашением http://www . mathnet.ru/rus/agreement

Параметры загрузки:

IP : 35.173 .137 .237

26 апреля 2023 г., 16:32:26

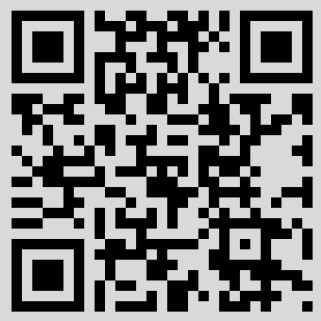




\section{НЕКОТОРЫЕ АСИМПТОТИЧЕСКИЕ ФОРМУЛЫ ДЛЯ ГАУССОВСКОЙ МЕРЫ БОГОЛЮБОВА}

Рассмотрены вопросы интегрирования по мере Боголюбова в пространстве непрерывных функций. Получены асимптотические формулы для одного класса функциональных интегралов типа Лапласа, взятых по мере Боголюбова. Также доказаны родственные асимптотические результаты о больших уклонениях для меры Боголюбова. В качестве основного функционала взята $L^{p}$-норма. Установлена гёльдеровость порядка $\gamma<1 / 2$ боголюбовских траекторий.

Ключевые слова: мера Боголюбова, метод Лапласа в банаховом пространстве.

\section{1. ВВЕДЕНИЕ И ФОРМУЛИРОВКА ОСНОВНЫХ РЕЗУЛЬТАТОВ}

В статьях [1]-[3] введена и изучена гауссовская мера Боголюбова, имеющая важное значение в теории статистического равновесия квантовых систем (см. [4]). Гауссовский процесс Боголюбова - это стационарный гауссовский процесс $\xi(t), t \in[0, \beta]$, с нулевым средним и ковариационной функцией

$$
B(t-s) \equiv B(t, s):=\mathbf{E} \xi(t) \xi(s)=\frac{1}{2 m \omega \operatorname{sh} \frac{\beta \omega}{2}} \operatorname{ch}\left(\omega|t-s|-\frac{\beta \omega}{2}\right), \quad t, s \in[0, \beta] .
$$

Здесь и ниже $\mathbf{E}$ - оператор математического ожидания, взятого относительно вероятности $\mathbf{P}, m>0$ - масса частицы, $\beta>0$ - обратная температура, $\omega>0$ собственная частота гармонического осциллятора [2].

Согласно общему результату из теории гауссовских процессов [5] процесс Боголюбова $\xi(t)$ имеет почти наверное (п.н.) непрерывные траектории на отрезке $[0, \beta]$. Более того, в силу теоремы Беляева [6] из разложения ковариационной функции $B(h)$ при $h \rightarrow 0$ следует, что траектории процесса $\xi(t)$ обладают свойством непрерывности по Гёльдеру порядка $\gamma<1 / 2$ (см. также [5]). Напомним, что в работе [3] доказано, что боголюбовские траектории не обладают свойством непрерывности по Гёльдеру порядка $\gamma>1 / 2$. Различные вопросы теории гауссовских процессов рассмотрены в книгах [5], [7]-[12].

* Московский государственный университет им. М. В. Ломоносова. E-mail:vfatalov@nw.math.msu.ru 
Используя формулу (1.1), легко находим, что $\mathbf{E}[\xi(\beta)-\xi(0)]^{2}=0$, следовательно, $\xi(0)=\xi(\beta)$ п.н. Учитывая вышеизложенное, заключаем, что траектории гауссовского процесса Боголюбова принадлежат п.н. пространству $C^{0}[0, \beta]$ непрерывных на отрезке $[0, \beta]$ функций $x(t)$, удовлетворяющих условию $x(0)=x(\beta)$, с равномерной метрикой. Распределение $\mu_{\mathrm{B}}$ процесса $\xi(t)$ в пространстве $C^{0}[0, \beta]$ называется гауссовской мерой Боголюбова.

Сведения о распределениях случайных процессов в функциональных пространствах имеются в книгах [7], [8]; теория гауссовских мер изложена в [7], [8], [10]-[12].

В статье [2] на основе метода работ [13] вычислены приближенно некоторые функциональные интегралы по мере Боголюбова. Приближенные формулы в [2] представляют собой квадратурные формулы, которые точны для функциональных многочленов. В дополнение к результатам работы [2] в настоящей статье мы получим асимптотические формулы для одного класса функциональных интегралов типа Лапласа, взятых по мере Боголюбова. Также мы докажем родственные асимптотические результаты о больших уклонениях для меры Боголюбова. Отметим, что в работах [14]-[18] описаны разнообразные важные задачи теории вероятностей и математической физики, связанные с вычислением асимптотик функциональных интегралов типа Лапласа и вероятностей больших уклонений.

Перейдем к точной формулировке полученных результатов.

Теорема 1. Пусть задано число $0<\alpha<2$. Для $p>0$ положим

$$
q_{0}=q_{0}(p, \alpha)=\frac{2-\alpha}{2}\left(\frac{\alpha}{m \omega^{2}} \beta^{(2-p) / p}\right)^{\alpha /(2-\alpha)}>0 .
$$

Справедливы следующие утверждения.

1. Предположим, что фиксировано число $0<p<2$. Тогда при $u \rightarrow \infty$ выполнено асимптотическое соотношение

$$
\int_{C^{0}[0, \beta]} \exp \left\{u\left(\int_{0}^{\beta}|x(t)|^{p} d t\right)^{\alpha / p}\right\} d \mu_{\mathrm{B}}(x)=d_{0} \exp \left\{q_{0} u^{2 /(2-\alpha)}\right\}(1+o(1)),
$$

где

$$
d_{0}=d_{0}(p, \alpha)=\frac{2 \sqrt{2-p} \operatorname{sh} \frac{\beta \omega}{2}}{\sqrt{2-\alpha} \operatorname{sh}\left(\frac{\beta \omega}{2} \sqrt{2-p}\right)}>0 .
$$

2. При $и \rightarrow \infty$ имеет место асимптотическое соотношение

$$
\begin{aligned}
& \int_{C^{0}[0, \beta]} \exp \left\{u\left(\int_{0}^{\beta} x^{2}(t) d t\right)^{\alpha / 2}\right\} d \mu_{\mathrm{B}}(x)= \\
& \quad=\exp \left\{\frac{2-\alpha}{2}\left(\frac{\alpha}{m \omega^{2}}\right)^{\alpha /(2-\alpha)} u^{2 /(2-\alpha)}\right\} \frac{4 \operatorname{sh} \frac{\beta \omega}{2}}{\beta \omega \sqrt{2-\alpha}}(1+o(1)) .
\end{aligned}
$$

3. Пусть фиксировано число $p>2$ такое, что

$$
p \neq 2+\frac{4 \pi^{2} n^{2}}{\beta^{2} \omega^{2}}, \quad n=1,2, \ldots
$$


Тогда при $и \rightarrow \infty$ выполнено асимптотическое соотношение

$$
\int_{C^{0}[0, \beta]} \exp \left\{u\left(\int_{0}^{\beta}|x(t)|^{p} d t\right)^{\alpha / p}\right\} d \mu_{\mathrm{B}}(x)=d_{1} \exp \left\{q_{0} u^{2 /(2-\alpha)}\right\}(1+o(1)),
$$

где

$$
d_{1}=d_{1}(p, \alpha)=\frac{2 \sqrt{p-2} \operatorname{sh} \frac{\beta \omega}{2}}{\sqrt{2-\alpha}\left|\sin \left(\frac{\beta \omega}{2} \sqrt{p-2}\right)\right|}>0 .
$$

ТЕОРема 2. Пусть фиксированъ числа $р>0 u 0<\alpha<2$, имеющие следующий вид:

$$
p=\frac{2 k_{1}-1}{2 k_{2}-1}, \quad \alpha=\frac{2 k_{3}-1}{2 k_{4}-1}, \quad \text { где } \quad k_{j}=1,2, \ldots, \quad j=1,2,3,4
$$

пусть при этом выполнено (1.4). Тогда при $u \rightarrow \infty$ справедливо асимптотическое соотношение

$$
\begin{aligned}
& \int_{C^{0}[0, \beta]} \exp \left\{u\left(\int_{0}^{\beta} x^{p}(t) d t\right)^{\alpha / p}\right\} d \mu_{\mathrm{B}}(x)= \\
& =\frac{1}{2} \int_{C^{0}[0, \beta]} \exp \left\{u\left(\int_{0}^{\beta}|x(t)|^{p} d t\right)^{\alpha / p}\right\} d \mu_{\mathrm{B}}(x)(1+o(1)) .
\end{aligned}
$$

Далее асимптотика вероятности из правой части последнего равенства вычисляется при помощи теоремы 1. Пусть

$$
\|x\|_{p}:=\left(\int_{0}^{\beta}|x(t)|^{p} d t\right)^{1 / p}, \quad p>0
$$

Как известно, при $p \geqslant 1$ функционал $\|x\|_{p}$ является нормой в банаховом пространстве $L^{p}[0, \beta]$ функций, $p$-я степень которых интегрируема на $[0, \beta]$.

ЗАмечАниЕ 1 . Ограничение $\alpha<2$ в теореме 1 есть следствие известной теоремы Ландау-Маркуса-Шеппа-Ферника об экспоненциальной интегрируемости квадрата нормы гауссовского случайного элемента, см. [8], [10]-[12], [15]. Согласно этой теореме, если $p \geqslant 1$, то $\mathbf{E} e^{u\|\xi\|_{p}^{\alpha}}<+\infty$ при $\alpha<2, u>0$ или при $\alpha=2, u<c_{p}$; $\mathbf{E} e^{u\|\xi\|_{p}^{\alpha}}=+\infty$ при $\alpha>2, u>0$ или при $\alpha=2, u \geqslant c_{p}$, где $c_{p}>0$ - некоторое вполне конкретное число. Так, $c_{2}=m \omega^{2} / 2$, см. предложение 1 ниже.

ЗАмЕчАниЕ 2. Значения чисел $p>0$ и $0<\alpha<2$ из теоремы 2 подобраны так, что для любого действительного числа $r$ определены однозначно степени $r^{p}, r^{\alpha / p}$, принимающие действительные значения. При этом выполнены равенства

$$
(-1)^{p}=-1, \quad\left(\int_{0}^{\beta}(-\xi(t))^{p} d t\right)^{\alpha / p}=-\left(\int_{0}^{\beta} \xi^{p}(t) d t\right)^{\alpha / p} .
$$

ЗАМЕчАниЕ 3 . Для чисел $p>2$ вида

$$
p=2+\frac{4 \pi^{2} n^{2}}{\beta^{2} \omega^{2}}, \quad n=1,2, \ldots,
$$


асимптотическое поведение вероятности из левой части формулы (1.5) нужно исследовать отдельно, возникает так называемый вырожденный случай (см. теорему 2.3 в работе [16]). Логарифмическая асимптотика имеет тот же вид: для чисел вида (1.9) и при $0<\alpha<2$ выполнено асимптотическое соотношение

$$
\lim _{u \rightarrow \infty} \frac{1}{u^{2 /(2-\alpha)}} \ln \mathbf{E} \exp \left\{u\left(\int_{0}^{\beta}|\xi(t)|^{p} d t\right)^{\alpha / p}\right\}=q_{0},
$$

где $q_{0}$ задано в (1.2).

Для чисел $p>2$ и $0<\alpha<2$, удовлетворяющих соотношениям (1.6) и (1.9), справедлива та же логарифмическая асимптотика:

$$
\lim _{u \rightarrow \infty} \frac{1}{u^{2 /(2-\alpha)}} \ln \mathbf{E} \exp \left\{u\left(\int_{0}^{\beta} \xi^{p}(t) d t\right)^{\alpha / p}\right\}=q_{0}
$$

В пограничном гильбертовом случае $\alpha=p=2$ возможны точные вычисления.

ПРЕДЛОЖЕНИЕ 1 (СЛУЧАЙ $\alpha=p=2$ ). Справедливљ равенства

$$
\int_{C^{0}[0, \beta]} \exp \left\{u \int_{0}^{\beta} x^{2}(t) d t\right\} d \mu_{\mathrm{B}}(x)=\frac{\operatorname{sh} \frac{\beta \omega}{2}}{\operatorname{sh}\left(\frac{\beta \omega}{2} \sqrt{\omega^{2}-\frac{2 u}{m}}\right)}, \quad \text { ecлu } \quad u<\frac{m \omega^{2}}{2} ;
$$

$\mathbf{E} \exp \left\{u \int_{0}^{\beta} \xi^{2}(t) d t\right\} \equiv \int_{C^{0}[0, \beta]} \exp \left\{u \int_{0}^{\beta} x^{2}(t) d t\right\} d \mu_{\mathrm{B}}(x)=+\infty, \quad$ если $\quad u \geqslant \frac{m \omega^{2}}{2}$.

Первое равенство в предложении доказано в работе [2], второе равенство вытекает из упомянутой выше теоремы Ландау-Маркуса-Шеппа-Ферника.

Результаты о больших уклонениях для меры Боголюбова в $L^{p}$-норме (1.8) имеют следующий вид.

Теорема 3. 1. Пусть фиксировано число $0<p<2$. Тогда при $u \rightarrow \infty$ выполнено асимптотическое соотношение

$$
\begin{aligned}
\mathbf{P}\left\{\int_{0}^{\beta}|\xi(t)|^{p} d t>u^{p}\right\} & \equiv \mu_{\mathrm{B}}\left\{x: \int_{0}^{\beta}|x(t)|^{p} d t>u^{p}\right\}= \\
& =\exp \left\{-\frac{m \omega^{2}}{2} \beta^{(p-2) / p} u^{2}\right\} \frac{d_{2}}{u}(1+o(1))
\end{aligned}
$$

где

$$
d_{2}=d_{2}(p)=\frac{\sqrt{2(2-p)} \beta^{(2-p) / 2 p} \operatorname{sh} \frac{\beta \omega}{2}}{\omega \sqrt{\pi m} \operatorname{sh}\left(\frac{\beta \omega}{2} \sqrt{2-p}\right)}>0 .
$$

2. При $и \rightarrow \infty$ справедливо асимптотическое соотношение

$$
\begin{aligned}
\mathbf{P}\left\{\int_{0}^{\beta} \xi^{2}(t) d t>u^{2}\right\} & \equiv \mu_{\mathrm{B}}\left\{x: \int_{0}^{\beta} x^{2}(t) d t>u^{2}\right\}= \\
& =\exp \left\{-\frac{m \omega^{2}}{2} u^{2}\right\} \frac{2 \sqrt{2} \operatorname{sh} \frac{\beta \omega}{2}}{u \beta \omega^{2} \sqrt{\pi m}}(1+o(1)) .
\end{aligned}
$$

5 Теоретическая и математическая физика, т. 157, № 2, 2008 г. 
3. Пусть фиксировано число $p>2$ такое, что выполнено условие невырожденности (1.4). Тогда при $и \rightarrow \infty$ имеет место асимптотическое соотношение

$$
\begin{aligned}
\mathbf{P}\left\{\int_{0}^{\beta}|\xi(t)|^{p} d t>u^{p}\right\} & \equiv \mu_{\mathrm{B}}\left\{x: \int_{0}^{\beta}|x(t)|^{p} d t>u^{p}\right\}= \\
& =\exp \left\{-\frac{m \omega^{2}}{2} \beta^{(p-2) / p} u^{2}\right\} \frac{d_{3}}{u}(1+o(1))
\end{aligned}
$$

где

$$
d_{3}=d_{3}(p)=\frac{\sqrt{2(p-2)} \beta^{(2-p) / 2 p} \operatorname{sh} \frac{\beta \omega}{2}}{\omega \sqrt{\pi m}\left|\sin \left(\frac{\beta \omega}{2} \sqrt{p-2}\right)\right|}>0 .
$$

Теорема 4. Пусть биксировано число $p>0$, имеющее вид, заданный в (1.6); пусть при этом выполнено условие (1.4). Тогда при $u \rightarrow \infty$ справедливо асимптотическое соотношение

$$
\mu_{\mathrm{B}}\left\{\int_{0}^{\beta} x^{p}(t) d t>u^{p}\right\}=\frac{1}{2} \mu_{\mathrm{B}}\left\{\int_{0}^{\beta}|x(t)|^{p} d t>u^{p}\right\}(1+o(1)) .
$$

Ясно, что асимптотику вероятности из правой части формулы (1.17) можно вычислить, применяя теорему 2.

ЗАмЕчАниЕ 4. Здесь также для чисел $p>2$ вида (1.9) возникает вырожденный случай (см. п. 3 леммы 8 ниже), поэтому для указанных значений $p$ асимптотическое поведение вероятности из левой части формулы (1.15) нужно исследовать отдельно. Логарифмическая асимптотика имеет тот же вид: для чисел $p$ вида (1.9)

$$
\lim _{u \rightarrow \infty} \frac{1}{u^{2}} \ln \mu_{\mathrm{B}}\left\{\int_{0}^{\beta}|x(t)|^{p} d t>u^{p}\right\}=-\frac{m \omega^{2}}{2} \beta^{(p-2) / p} .
$$

Для чисел $p>2$, удовлетворяющих соотношениям (1.6) и (1.9), справедлива та же логарифмическая асимптотика:

$$
\lim _{u \rightarrow \infty} \frac{1}{u^{2}} \ln \mu_{\mathrm{B}}\left\{\int_{0}^{\beta} x^{p}(t) d t>u^{p}\right\}=-\frac{m \omega^{2}}{2} \beta^{(p-2) / p} .
$$

ЗАмЕчАНИЕ 5. Согласно общему свойству гауссовских процессов с нулевым средним процесс $-\xi(t), t \in[0, \beta]$, также представляет собой процесс Боголюбова. Поэтому в условиях теорем 2 и 4 выполнены соответственно равенства

$$
\begin{aligned}
& \int_{C^{0}[0, \beta]} \exp \left\{u\left(\int_{0}^{\beta} x^{p}(t) d t\right)^{\alpha / p}\right\} d \mu_{\mathrm{B}}(x)= \\
& =\int_{C^{0}[0, \beta]} \exp \left\{-u\left(\int_{0}^{\beta} x^{p}(t) d t\right)^{\alpha / p}\right\} d \mu_{\mathrm{B}}(x), \\
& \mu_{\mathrm{B}}\left\{\int_{0}^{\beta} x^{p}(t) d t>u^{p}\right\}=\mu_{\mathrm{B}}\left\{\int_{0}^{\beta} x^{p}(t) d t<-u^{p}\right\} .
\end{aligned}
$$

Следовательно, к выражениям из правых частей равенств (1.20) и (1.21) применимы, соответственно, формулы (1.7) и (1.17). 
Аналоги теорем 1 и 3 для винеровской меры доказаны в работах [19], [20] (см. также [21]). Отметим, что аналоги теорем 1-4 можно доказать и в случае, когда вместо $L^{p}$-нормы взят другой достаточно гладкий интегральный функционал (см. теоремы 5-7 ниже).

В заключение этого раздела опишем интересную связь между мерами Винера и Боголюбова. Сделать это удобно в терминах теории случайных процессов. Пусть $w(t), t \geqslant 0$, обозначает винеровский процесс, или броуновское движение, $w(0)=0$ п.н. Напомним, что винеровский процесс $w(t)$ - это п.н. непрерывный гауссовский процесс с нулевым средним и ковариационной функцией $\mathbf{E} w(t) w(s)=\min (t, s)$ (см., например, [10], [22], [23]). Исходя из процесса Боголюбова $\xi(t)$ определим случайный процесс

$$
\eta(t)=\sqrt{m}(\xi(t)-\xi(0))+\sqrt{m} \omega \int_{0}^{t} \xi(s) d s, \quad t \in[0, \beta] .
$$

ПреДЛОЖениЕ 2 [3]. Распределение процесса $\eta(t), t \in[0, \beta]$, совпадает с pacnpeделением винеровского процесса $w(t), t \in[0, \beta]$.

Предложение 2 фактически доказано в статье [3], где оно, однако, не сформулировано в явном виде. Доказательство предложения 2 простое. В самом деле, п.н. непрерывный случайный процесс $\eta(t)$ представляет собой результат линейного преобразования (1.22) над гауссовским процессом $\xi(t)$, следовательно, процесс $\eta(t)$ является гауссовским. Из формулы (1.22) получаем, что $\mathbf{E} \eta(t)=0, t \in[0, \beta]$. Несложный подсчет с использованием соотношений (1.1) и (1.22) показывает, что $\mathbf{E} \eta(t) \eta(s)=\min (t, s)$ для всех $t, s \in[0, \beta]$. Таким образом, ковариационная функция гауссовского процесса $\eta(t)$ совпадает с ковариационной функцией винеровского процесса $w(t)$. Отсюда, очевидно, вытекает утверждение предложения 2.

Отметим, что из предложения 2 и теоремы 3.5 книги [10] непосредственно следует теорема 3 статьи [3].

\section{2. МЕТОД ЛАПЛАСА ДЛЯ ГАУССОВСКИХ МЕР В СЛУЧАЕ ГЛАДКИХ ФУНКЦИОНАЛОВ}

В работах [18], [19], [24] был построен эффективный метод Лапласа, предназначенный для вычисления точных асимптотик функциональных интегралов типа Лапласа и вероятностей больших уклонений в случае общих гауссовских мер в банаховом пространстве. Сформулируем результат, лежащий в основе метода Лапласа для гауссовских интегралов в банаховом пространстве.

Пусть $(E,\|\cdot\|)$ - действительное сепарабельное банахово пространство, $\mathcal{E}$ - борелевская $\sigma$-алгебра в $E, E^{*}$ - сопряженное к $E$ пространство, $\langle\cdot, \cdot\rangle-$ линейная форма, устанавливающая двойственность между $E$ и $E^{*}$. Пусть $P_{A}$ - гауссовская мера, определенная на $\mathcal{E}$ и имеющая нулевое среднее значение и инъективный ковариационный оператор $A: E^{*} \rightarrow E$. Напомним, что ковариационный оператор $A$ является симметричным, т.е. $\langle A x, y\rangle=\langle A y, x\rangle$ для всех $x, y \in E^{*}$, положительным, т.е. $\langle A y, y\rangle \geqslant 0$ для всех $y \in E^{*}$, и ядерным [12]. Инъективность оператора $A$ означает, что $\langle A y, y\rangle>0$ для всех $0 \neq y \in E^{*}$. 
Обозначим первую и вторую производные по Фреше функционала $S: E \rightarrow \mathbb{R}$ в точке $x$ через $S^{\prime}(x)$ и $S^{\prime \prime}(x)$, необходимые определения можно найти в книгах [25], [26]. Мы будем предполагать, что производные $S^{\prime}(x)$ и $S^{\prime \prime}(x)$ можно рассматривать как ограниченные линейные операторы из $E$ в $\mathbb{R}$ и из $E$ в $E^{*}$ соответственно, при этом будем использовать обозначения

$$
S^{\prime}(x)[h]=\left\langle h, S^{\prime}(x)\right\rangle, \quad S^{\prime \prime}(x)[h, v]=\left\langle h, S^{\prime \prime}(x) v\right\rangle, \quad h, v \in E .
$$

Обозначим также $\operatorname{Dom} T$ область определения оператора $T, \operatorname{Ran} T$ - область значений оператора $T, \operatorname{Ker} T:=\{x \in E: T x=0\}$ - ядро оператора $T$, заданного на $E$; пусть $I$ - тождественный оператор в $E$. Через $T^{-1}$ будем обозначать обратный оператор к оператору $T$. Везде ниже произведение операторов означает их композицию. Операторная норма обозначается также через $\|\cdot\|$.

Пусть $\left(H_{A},\|\cdot\|_{A}\right)$ обозначает гильбертово пространство, ассоциированное с ковариационным оператором $A$, т.е. пополнение области значений $\operatorname{Ran} A$ оператора $A$ относительно предгильбертовой нормы $\|A y\|_{A}:=\sqrt{\langle A y, y\rangle}, y \in E^{*}$, см. [12]. Скалярное произведение в $H_{A}$ будем записывать в виде $\langle\cdot, \cdot\rangle_{A}$.

\section{1. Точные асимптотики для гауссовских интегралов типа Лапласа.} Приведем теорему, с помощью которой можно вычислять точные асимптотики средних из формул (1.3) и (1.7). Предположим, что выполнены следующие условия.

D1. Пусть $f$ и $F$ - непрерывные действительные функционалы, заданные на $E$, такие, что для некоторых постоянных $b_{1}>0, b_{2} \geqslant 0,0 \leqslant b_{3}<(2\|A\|)^{-1}, b_{4} \geqslant 0$ справедливы неравенства

$$
|f(x)| \leqslant b_{1} e^{b_{2}\|x\|^{2}}, \quad F(x) \geqslant-b_{3}\|x\|^{2}-b_{4}, \quad x \in E .
$$

D2. Функционал $\Lambda(x):=F(x)+\left\langle x, A^{-1} x\right\rangle / 2$ достигает минимума на $\operatorname{Dom} A^{-1}$ в конечном числе точек $x^{1}, x^{2}, \ldots, x^{m}$. Предположим, что $\inf _{x \in H_{A}}\left[F(x)+\|x\|_{A}^{2} / 2\right]$ также достигается только в точках $x^{1}, x^{2}, \ldots, x^{m}$.

D3. В пространстве $E$ существуют такие окрестности $U_{i}$ точек $x^{i}$, что функционал $F$ трижды дифференцируем по Фреше в точках множеств $U_{i}$, при этом $f\left(x^{i}\right) \neq 0$, $i=1,2, \ldots, m$.

D4. Для всех $i=1,2, \ldots, m$ собственные значения $\gamma_{i k}, k=1,2, \ldots$, ядерного оператора $A F^{\prime \prime}\left(x^{i}\right): E \rightarrow E$ таковы, что

$$
\operatorname{det} R_{i}:=\prod_{k=1}^{\infty}\left(1+\gamma_{i k}\right), \quad R_{i}=I+A F^{\prime \prime}\left(x^{i}\right): E \rightarrow E,
$$

конечен и не равен нулю.

Сведения о детерминантах, связанных с ядерными операторами в гильбертовом и банаховом пространствах имеются в книгах [15], [27]-[29].

Теорема 5. Пусть $P_{A}$ - гауссовская мера в Е с нулевым средним и инбективным ковариационным оператором $A$. Пусть выполнены условия D1-D4. Тогда $\operatorname{det} R_{i}>0$, имеют место равенства

$$
x^{i}=-A F^{\prime}\left(x^{i}\right), \quad i=1,2, \ldots, m,
$$


и при $и \rightarrow \infty$ справедливо асимптотическое соотношение

$$
\int_{E} f(x) e^{-u^{2} F(x)} d P_{A}(u x)=e^{-u^{2} \Lambda\left(x^{1}\right)} \sum_{i=1}^{m} f\left(x^{i}\right) \operatorname{det}^{-1 / 2} R_{i}(1+o(1)) .
$$

Теорема 5 представляет собой банаховозначный вариант известного результата Эллиса и Розен [16] об асимптотическом разложении гауссовского интеграла типа Лапласа в гильбертовом пространстве (см. также [18]).

Отметим, что условие D3, использующееся в теореме 5, является значительно более слабым, чем соответствующее условие из [16] о бесконечной дифференцируемости по Фреше функционалов $f$ и $F$ на всем пространстве. Ограничение на $F$, содержащееся в условии D1, обеспечивает конечность интеграла в левой части формулы (2.1) - это следует из упомянутой выше теоремы Ландау-Маркуса-Шеппа-Ферника. Примеры применения теоремы 5 для функционала $F(x)=\left(\int_{0}^{1}|x(t)|^{p} d t\right)^{\alpha / p}$ при $p>0,0<\alpha<2$ в случае винеровского процесса и броуновского моста даны в работе [20].

\section{2. Точные асимптотики больших уклонений для гауссовских мер.} Приведем теперь результат, на основе которого выводятся утверждения теорем 3 и 4. Здесь мы рассмотрим более простой случай, когда $E$ представляет собой сепарабельное гильбертово пространство $H$ с нормой $\|\cdot\|$ и скалярным произведением $\langle\cdot, \cdot\rangle$. Предположим, что выполнены следующие условия.

C1. Борелевское множество $D$ замкнуто и может быть представлено в виде $D=\{x \in H: Q(x) \geqslant 0\}$, где $Q(x)$ - непрерывный действительный функционал такой, что либо $D$, либо множество $H \backslash D$ представимо в виде конечного объединения непересекающихся выпуклых множеств из $H$. Пусть $D$ имеет непустую внутренность и не содержит нулевого элемента из $H$.

C2. Квадратичная форма $\left\langle x, A^{-1} x\right\rangle$ достигает своего минимума на $D \cap \operatorname{Dom} A^{-1}$ в конечном числе точек $x^{1}, x^{2}, \ldots, x^{m}$ из границы множества $D$. Предположим, что $\inf _{x \in D \cap H_{A}}\|x\|_{A}^{2}$ также достигается только в точках $x^{1}, x^{2}, \ldots, x^{m}$.

C3. В гильбертовом пространстве $H$ существуют такие окрестности $U_{i}$ точек $x^{i}$, что функционал $Q$ трижды дифференцируем по Фреше в точках множеств $U_{i}$, причем $\left\langle x^{i}, Q^{\prime}\left(x^{i}\right)\right\rangle \neq 0, i=1,2, \ldots, m$.

C4. Для всех $i=1,2, \ldots, m$ собственные значения $\gamma_{i k}, k=1,2, \ldots$, ядерного оператора $A Q^{\prime \prime}\left(x^{i}\right): H \rightarrow H$ таковы, что не равен нулю

$$
\operatorname{det} R_{i}:=\prod_{k=1}^{\infty}\left(1-\lambda_{i} \gamma_{i k}\right)
$$

где $R_{i}=I-\lambda_{i} A Q^{\prime \prime}\left(x^{i}\right): H \rightarrow H$ и

$$
\lambda_{i}=\frac{\left\langle x^{1}, A^{-1} x^{1}\right\rangle}{\left\langle x^{i}, Q^{\prime}\left(x^{i}\right)\right\rangle}>0 .
$$

Из условия $\mathbf{C 4}$ вытекает, что операторы $R_{i}$ являются инъективными, Ker $R_{i}=$ $\{0\}, i=1,2, \ldots, m$. Следовательно, согласно альтернативе Фредгольма [30] 
$\operatorname{Ran} R_{i}=H$, и существует обратный оператор $R_{i}^{-1}$, определенный на всем $H . \mathrm{B}$ частности, $x^{i} \in \operatorname{Dom} R_{i}^{-1}, i=1,2, \ldots, m$.

Теорема 6. Пусть $P_{A}$ - гауссовская мера в гильбертовом пространстве $H$, имеющая нулевое среднее и ингективный ковариационный оператор А. Пусть выполнены условия C1-C4. Тогда имеют место равенства

$$
x^{i}=\lambda_{i} A Q^{\prime}\left(x^{i}\right), \quad i=1,2, \ldots, m,
$$

и при $и \rightarrow \infty$ справедливо асимптотическое соотношение

$$
P_{A}(u D)=\exp \left\{-\frac{u^{2}}{2}\left\langle x^{1}, A^{-1} x^{1}\right\rangle\right\} \frac{1}{u \sqrt{2 \pi}} \sum_{i=1}^{m}\left|\operatorname{det} R_{i}\left\langle R_{i}^{-1} x^{i}, A^{-1} x^{i}\right\rangle\right|^{-1 / 2}(1+o(1)) .
$$

Теорема 6 доказана в работе [24], банаховозначный вариант изложен в статьях [18], [19]. Условия C2-C4 в теореме 6 записаны в несколько иной форме, более точной, чем соответствующие условия в работах [18], [19], [24].

Существует несколько модификаций теоремы 6, в которых используются иные условия. Приведем две такие модификации, довольно часто применяющиеся на практике. Напомним, что функиионал действия гауссовской меры $P_{A}$ в пространстве $H$ определяется следующим образом (см. [8], [18], [31]):

$$
I_{A}(x)=\left\{\begin{array}{lll}
\frac{1}{2}\|x\|_{A}^{2}, & \text { если } & x \in H_{A}, \\
+\infty, & \text { если } & x \in H \backslash H_{A} .
\end{array}\right.
$$

Введем следующее условие.

$\widetilde{\mathbf{C 1}}$. Борелевское множество $D$ замкнуто в $H$ и может быть представлено в виде $D=\{x \in H: Q(x) \geqslant 0\}$, где $Q(x)$ - непрерывный действительный функционал. Пусть $D$ имеет непустую внутренность Int $D=\{x \in H: Q(x)>0\}$ и не содержит нулевого элемента из $H$. Предположим, что выполнено равенство

$$
\inf _{x \in \operatorname{Int} D} I_{A}(x)=\inf _{x \in D} I_{A}(x) .
$$

Первая модификация теоремы 6, связанная с освобождением от требования выпуклости в условии $\mathbf{C 1}$, имеет следующий вид.

Теорема 6'. Пусть $P_{A}$ - гауссовская мера в гильбертовом пространстве $H$, имеющая нулевое среднее и инбективный ковариационный оператор А. Пусть выполнены условия $\widetilde{\mathbf{C 1}}, \mathbf{C 2}-\mathbf{C 4}$. Тогда имеют место равенства (2.4) и справедливо асимптотическое соотношение (2.5).

Сформулируем еще одну модификацию теоремы 6, связанную с вырождением (обращением в нуль) $\operatorname{det} R_{i}$, заданного в (2.2). В этом случае асимптотика, стоящая в правой части формулы (2.5), заменяется на иное выражение.

Теорема 7. Пусть $P_{A}$ - гауссовская мера в гильбертовом пространстве $H$, имеющая нулевое среднее и инбективный ковариационный оператор А. Пусть выполнены условия $\mathbf{C 1 - C 3 ~ и ~ с л е д у ю щ е е ~ у с л о в и е . ~}$ 
$\widetilde{\mathbf{C 4}}$. Для каждого $i=1,2, \ldots, m$ ядро оператора $R_{i}=I-\lambda_{i} A Q^{\prime \prime}\left(x^{i}\right)$ представляет собой одномерное подпространство в $H$, натянутое на вектор $e_{i} \in H$, причем $\left\|e_{i}\right\|=1,\left\langle e_{i}, x^{i}\right\rangle \neq 0 u\left\langle e_{i}, A^{-1} x^{i}\right\rangle \neq 0$.

Тогда имеют место равенства (2.4) и при $u \rightarrow \infty$ справедливо асимптотическое соотношение

$$
P_{A}(u D)=\exp \left\{-\frac{u^{2}}{2}\left\langle x^{1}, A^{-1} x^{1}\right\rangle\right\} \frac{1}{u \sqrt{2 \pi}} \sum_{i=1}^{m}\left|\left\langle e_{i}, x^{i}\right\rangle\left\langle e_{i}, A^{-1} x^{i}\right\rangle \operatorname{det} R_{i 0}\right|^{-1 / 2}(1+o(1)) .
$$

Здесъ

$$
\operatorname{det} R_{i 0}:=\prod_{k=1}^{\infty}\left(1-\lambda_{i} \gamma_{i k}\right)
$$

где $\gamma_{i k}, k=1,2, \ldots,-$ собственнъе значения ядерного оператора $A Q^{\prime \prime}\left(x^{i}\right): H \rightarrow H$ maкuе, что $\gamma_{i k} \neq 1 / \lambda_{i}$.

\section{3. ВЫБОР ГИЛЬБЕРТОВА ФУНКЦИОНАЛЬНОГО ПРОСТРАНСТВА}

Мы докажем теоремы 1 и 2, применяя теорему 5, доказательство теорем 3 и 4 проведем при помощи теорем $6,6^{\prime}, 7$. Нам удобней сначала доказать теоремы 3 и 4 .

Возьмем в качестве гильбертова пространства $H$ обычное гильбертово пространство $L^{2} \equiv L^{2}[0, \beta]$ действительных измеримых функций на $[0, \beta]$, квадрат которых интегрируем, со скалярным произведением и нормой, имеющими следующий вид:

$$
\langle x, y\rangle=\int_{0}^{\beta} x(t) y(t) d t, \quad\|x\|=\left(\int_{0}^{\beta} x^{2}(t) d t\right)^{1 / 2}, \quad x, y \in L^{2} .
$$

Мы будем полагать, что гауссовская мера Боголюбова $\mu_{\mathrm{B}}$ задана в пространстве $L^{2}$, имея в виду, что эта мера сосредоточена на $C^{0}[0, \beta]$.

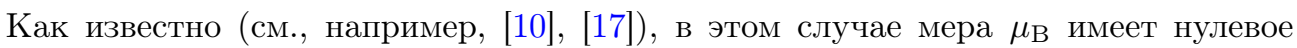
среднее и ковариационный оператор $A_{\mathrm{B}}: L^{2} \rightarrow L^{2}$ интегрального типа:

$$
A_{\mathrm{B}} x(t)=\int_{0}^{\beta} B(t, s) x(s) d s=\frac{1}{2 m \omega \operatorname{sh} \frac{\beta \omega}{2}} \int_{0}^{\beta} \operatorname{ch}\left(\omega|t-s|-\frac{\beta \omega}{2}\right) x(s) d s, \quad x \in L^{2} .
$$

Пусть $C^{2}[0, \beta]$ - множество дважды дифференцируемых на $[0, \beta]$ функций $x(t)$.

Лемма 1. Область значений ковариационного оператора $A_{\mathrm{B}}$, определенного в формуле (3.1), имеет вид

$$
\operatorname{Ran} A_{\mathrm{B}}=\left\{x \in C^{2}[0, \beta]: x(0)=x(\beta), x^{\prime}(0)=x^{\prime}(\beta)\right\} .
$$

Обратный оператор задается формулой

$$
\left[A_{\mathrm{B}}^{-1} y\right](t)=-m y^{\prime \prime}(t)+m \omega^{2} y(t), \quad y \in \operatorname{Ran} A_{\mathrm{B}}, \quad t \in[0, \beta] .
$$


ДокАзАтЕльство. Утверждения леммы получаются в результате двукратного дифференцирования по $t$ равенства $y(t)=A_{\mathrm{B}} x(t)$, (см., например, справочник [32]).

Лемма 2. Собственные числа $\left\{\nu_{n}\right\}_{n=-\infty}^{\infty}$ ковариационного оператора $A_{\mathrm{B}}$ имеют следующий вид:

$$
\nu_{n}=\frac{1}{m\left(\omega^{2}+\left(2 \pi \beta^{-1} n\right)^{2}\right)}, \quad n=0, \pm 1, \pm 2, \ldots,
$$

им соответствуют $L^{2}$-нормированные собственные функции

$$
\varphi_{n}(t)=\sqrt{\frac{1}{\beta}}\left\{\begin{array}{lll}
\sqrt{2} \sin \frac{2 \pi n t}{\beta}, & \text { если } & n<0 \\
1, & \text { еслu } & n=0 \\
\sqrt{2} \cos \frac{2 \pi n t}{\beta}, & \text { еслu } & n>0 .
\end{array}\right.
$$

ДокАзАтельство. Рассмотрим задачу на собственные числа для ядерного опеpaтора $A_{\mathrm{B}}$ :

$$
A_{\mathrm{B}} \varphi=\nu \varphi, \quad \varphi \in L^{2} .
$$

Поскольку оператор $A_{\mathrm{B}}$ является инъективным и положительным, в уравнении (3.6) параметр $\nu>0$. Согласно соотношениям (3.2), (3.3) уравнение (3.6) равносильно следующей краевой задаче на собственные числа:

$$
\begin{gathered}
\varphi^{\prime \prime}(t)+\left(\frac{1}{m \nu}-\omega^{2}\right) \varphi(t)=0, \quad t \in[0, \beta], \\
\varphi(0)=\varphi(\beta), \quad \varphi^{\prime}(0)=\varphi^{\prime}(\beta) .
\end{gathered}
$$

Используя справочник [33], заключаем, что решениями задачи (3.7) являются собственные числа (3.4) и собственные функции (3.5). Лемма доказана.

Отметим, что утверждение леммы 2 получено иным способом в статье [1], пояснения см. в [2].

\section{4. ДОКАЗАТЕЛЬСТВО ТЕОРЕМЫ 3}

Мы докажем теорему 3 , применяя теоремы $6,6^{\prime}$ и 7 , в которых будем полагать $H=L^{2}, P_{A}=\mu_{\mathrm{B}}, A=A_{\mathrm{B}}$. Пусть фиксировано число $p>0$. До определенного момента доказательство всех трех пунктов теоремы 3 проводится единым образом.

4.1. Выбор основного функционала. Возьмем в качестве функционала $Q$, участвующего в формулировках теорем $6,6^{\prime}, 7$ (см. формулу (2.4)), следующий функционал:

$$
G(x):=\int_{0}^{\beta}|x(t)|^{p} d t-1, \quad x \in L^{2} .
$$

Обозначим через $\mathcal{X}$ множество функций из $C^{0}[0, \beta]$, имеющих конечное число нулей. Очевидно, множество $\mathcal{X}$ плотно в $C^{0}[0, \beta]$, а следовательно, и в $L^{2}$. Отметим, что $0 \notin \mathcal{X}$ (здесь функция, тождественно равная нулю на $[0, \beta]$, также обозначена через 0$)$. 
Ниже мы используем определение производной по Фреше, данное в [25], где допускается, что рассматриваемый оператор определен лишь на некотором всюду плотном подмножестве банахова пространства; кроме того, мы полагаем, что он может принимать значения, равные $\pm \infty$ (см. определение 1 в [26]).

ЛЕмма 3. 1. Функиионал $G$ трижды дифферениируем по Фреше в каждой точке $x \in \mathcal{X}$ и имеет производные

$$
\begin{gathered}
G^{\prime}(x)=p|x(t)|^{p-1} \operatorname{sign} x(t), \\
G^{\prime \prime}(x)=p(p-1)|x(t)|^{p-2} \\
G^{\prime \prime \prime}(x)[h, g, v]=\int_{0}^{\beta} p(p-1)(p-2)|x(t)|^{p-3} \operatorname{sign} x(t) h(t) g(t) v(t) d t, \quad h, g, v \in L^{2} .
\end{gathered}
$$

2. Функиионал $G$ является выпуклым функционалом ${ }^{1)}$ при $p \geqslant 1$.

ДокАзАтельство. Утверждение 1 леммы получаем, применяя известные формулы для первых трех производных интегрального функционала (4.1) [26], [34]. Утверждение 2 леммы следует из выпуклости вниз функции $|x|^{p}$ при $p \geqslant 1$ и $x \in \mathbb{R}$. Отметим, что функционал (4.1) не является выпуклым вверх функционалом при $0<p<1$.

\section{2. Решение экстремальной задачи. Положим}

$$
D=\left\{x \in L^{2}: G(x) \geqslant 0\right\}
$$

В силу п. 2 леммы 3 множество $L^{2} \backslash D$ является выпуклым при $p \geqslant 1$. Очевидно, что множество (4.4) имеет непустую внутренность и не содержит нулевого элемента. Таким образом, множество (4.4) удовлетворяет условию $\mathbf{C 1}$ при $p \geqslant 1$. Можно показать, что при $0<p<1$ множество (4.4) удовлетворяет условию $\widetilde{\mathbf{C 1}}$.

Решим экстремальную задачу на минимум $\left\langle x, A_{\mathrm{B}}^{-1} x\right\rangle$ из условия $\mathbf{C 2}$ для множества $D$, определенного в (4.4), и ковариационного оператора $A_{\mathrm{B}}$, заданного в $(3.1)$. Для $p>0$ определим постоянную функцию

$$
x_{0}(t)=\beta^{-1 / p}, \quad t \in[0, \beta] .
$$

Лемма 4. Пусть $р>0$ фиксировано. Тогда минимум в экстремальной задаче

$$
\left\langle x, A_{\mathrm{B}}^{-1} x\right\rangle \rightarrow \inf , \quad x \in D \cap \operatorname{Dom} A_{\mathrm{B}}^{-1},
$$

равен $m \omega^{2} \beta^{(p-2) / p}$ и достигается на двух функциях $x_{0}(t) u-x_{0}(t)$, лежащих на гранище дD. Минимум $\inf _{x \in D \cap H_{A_{w}}}\|x\|_{A_{\mathrm{B}}}^{2}$ также достигается только в двух точ$\operatorname{\kappa ax} x_{0} u-x_{0}$.

\footnotetext{
1) Напомним, что определение и некоторые свойства выпуклых множеств и выпуклых функционалов в банаховом пространстве имеются в книгах [25], [26]. Выпуклые вниз функции часто называют просто выпуклыми.
} 
ДокАЗАТЕЛЬСтво. Покажем, что минимум в задаче (4.6) достижим. В силу леммы 2 максимальное собственное значение оператора $A_{\mathrm{B}}$ равно $\nu_{0}=1 / m \omega^{2}$. Следовательно, минимальное собственное значение оператора $A_{\mathrm{B}}^{-1}$ равно $m \omega^{2}$. Таким образом, согласно известному вариационному описанию собственных значений компактного симметричного оператора [35], [36] справедлива оценка $\left\langle x, A_{\mathrm{B}}^{-1} x\right\rangle \geqslant m \omega^{2}\|x\|^{2}$, $x \in L^{2}$. Из этой оценки и первой теоремы Вейерштрасса (см. теорему 9.2 и замечание 9.1 в [25]) следует, что минимум в задаче (4.6) достигается.

Решая экстремальную задачу (4.6) методом множителей Лагранжа [26], убеждаемся, что точка, доставляющая минимум в задаче (4.6), принадлежит границе, и для нее выполнено соотношение $x=\lambda A_{\mathrm{B}} G^{\prime}(x)$ (ср. с (2.4)). Таким образом, уравнение для экстремальной функции $x(t)$ согласно соотношениям (3.1) и (4.2) является нелинейным интегральным уравнением Хаммерштейна и имеет вид

$$
x(t)=\frac{\lambda p}{2 m \omega \operatorname{sh} \frac{\beta \omega}{2}} \int_{0}^{\beta} \operatorname{ch}\left(\omega|t-s|-\frac{\beta \omega}{2}\right)|x(s)|^{p-1} \operatorname{sign} x(s) d s, \quad t \in[0, \beta],
$$

где $\lambda>0$ - множитель Лагранжа, при этом выполнено условие связи

$$
\int_{0}^{\beta}|x(t)|^{p} d t=1
$$

Поскольку $x \in \operatorname{Ran} A_{\mathrm{B}}$, заключаем, учитывая (3.2), (3.3), что экстремальная функция дважды дифференцируема на $[0, \beta]$. Используя формулу (3.3) для обратного оператора, убеждаемся, что интегральное уравнение (4.7) эквивалентно следующей нелинейной краевой задаче:

$$
\begin{gathered}
x^{\prime \prime}(t)=\omega^{2} x(t)-\frac{\lambda p}{m}|x(t)|^{p-1} \operatorname{sign} x(t), \quad t \in[0, \beta], \\
x(0)=x(\beta), \quad x^{\prime}(0)=x^{\prime}(\beta) .
\end{gathered}
$$

Мы будем искать положительное решение задачи (4.9) с условием (4.8), т.е. будем полагать, что $x(t)>0, t \in[0, \beta]$. Можно показать, что знакопеременные решения задачи (4.8), (4.9) не доставляют минимума в экстремальной задаче (4.6).

Для положительного $x(t)$ уравнение в (4.9) и условие (4.8) принимают вид

$$
\begin{gathered}
x^{\prime \prime}(t)=\omega^{2} x(t)-\frac{\lambda p}{m} x^{p-1}(t), \quad t \in[0, \beta], \\
\int_{0}^{\beta} x^{p}(t) d t=1 .
\end{gathered}
$$

Записывая уравнение (4.10) в виде $d\left(x^{\prime}(t)\right)^{2}=\omega^{2} d x^{2}(t)-(2 \lambda / m) d x^{p}(t)$, приходим к следующему факту: если функция $x(t)$ удовлетворяет уравнению (4.10), то эта функция удовлетворяет также уравнению

$$
\left(x^{\prime}(t)\right)^{2}=\omega^{2} x^{2}(t)-\frac{2 \lambda}{m} x^{p}(t)+C, \quad t \in[0, \beta],
$$

при некоторой константе $C$. Учитывая это, несложно показать, что для заданного значения $\lambda>0$ задача (4.9) имеет единственное положительное решение 
$x_{0}(t) \equiv h>0$, которое является постоянной функцией. Используя условие связи (4.11), получаем $h=\beta^{-1 / p}$, следовательно, найденная положительная функция имеет вид (4.5). Подставляя функцию (4.5) в уравнение (4.10), получаем значение множителя Лагранжа

$$
\lambda_{0}=\frac{m \omega^{2}}{p} \beta^{(p-2) / p} .
$$

Учитывая вышеизложенное, приходим к выводу, что единственная положительная функция $x_{0}$, доставляющая минимум в задаче (4.6), имеет вид (4.5). Ясно, что в силу формулы $(4.11) x_{0}(t)$ принадлежит границе множества $D$. Очевидно, что отрицательная функция $-x_{0}(t)$ также доставляет минимум в задаче (4.6).

Используя формулы (3.3), (4.5), найдем минимальное значение $\left\langle x, A_{\mathrm{B}}^{-1} x\right\rangle$ :

$$
\left\langle x_{0}, A_{\mathrm{B}}^{-1} x_{0}\right\rangle=m \omega^{2} \int_{0}^{\beta} x_{0}^{2}(t) d t=m \omega^{2} \beta^{(p-2) / p} .
$$

Рассматривая гильбертово пространство $\left(H_{A_{\mathrm{B}}},\|\cdot\|_{A_{\mathrm{B}}}\right)$, ассоциированное с ковариа-

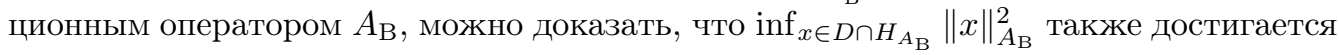
только в двух точках $x_{0}$ и $-x_{0}$. Лемма доказана.

Отметим, что при $p=2$ утверждение леммы 4 согласуется с леммой 2: функция (4.5) при $p=2$ совпадает с собственной функцией $\varphi_{0}(t)$ из формулы $(3.5)$, а минимальное значение $m \omega^{2}$ совпадает с $1 / \nu_{0}$.

4.3. Исследование спектра интегрального оператора $A_{\mathrm{B}} G^{\prime \prime}\left(x_{0}\right)$. Из лемм 3,4 следует, что при $p \geqslant 1$ гауссовская мера Боголюбова $\mu_{\mathrm{B}}$ и множество (4.4) удовлетворяют условиям C1-C3 с $m=2, x^{1}=x_{0}$ и $x^{2}=-x_{0}$. При $0<p<1$

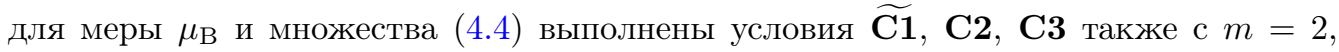
$x^{1}=x_{0}$ и $x^{2}=-x_{0}$. В обоих случаях в силу соотношений $(4.2),(4.12)$ для чисел $\lambda_{i}$ из формулы (2.3) справедливы равенства $\lambda_{1}=\lambda_{2}=\lambda_{0}$. Согласно формуле (4.3) имеет место равенство $G^{\prime \prime}\left(x_{0}\right)=G^{\prime \prime}\left(-x_{0}\right)$, поэтому достаточно исследовать спектр интегрального оператора $A_{\mathrm{B}} G^{\prime \prime}\left(x_{0}\right): L^{2} \rightarrow L^{2}$. Этот оператор является ядерным как композиция ядерного оператора $A_{\mathrm{B}}$ и ограниченного оператора $G^{\prime \prime}\left(x_{0}\right)[37]$. В силу формул (4.3) и (4.5) выполнено равенство

$$
G^{\prime \prime}\left(x_{0}\right)=p(p-1) \beta^{(2-p) / p} .
$$

Лемма 5. Пусть $p>0$ фиксировано. Тогда собственные числа $\left\{\gamma_{n}\right\}_{n=-\infty}^{\infty}$ ядерного интегрального оператора $A_{\mathrm{B}} G^{\prime \prime}\left(x_{0}\right)$ имеют следующий вид:

$$
\gamma_{n}=\frac{p(p-1) \beta^{(2-p) / p}}{m\left(\omega^{2}+\left(2 \pi \beta^{-1} n\right)^{2}\right)}, \quad n=0, \pm 1, \pm 2, \ldots .
$$

ДокАЗАТЕльство. Рассмотрим задачу на собственные числа для ядерного опеpaтора $A_{\mathrm{B}} G^{\prime \prime}\left(x_{0}\right)$ :

$$
A_{\mathrm{B}} G^{\prime \prime}\left(x_{0}\right) z=\gamma z, \quad z \in L^{2} .
$$

Эта задача согласно формуле (4.14) принимает следующий вид:

$$
p(p-1) \beta^{(2-p) / p} A_{\mathrm{B}} z=\gamma z, \quad z \in L^{2},
$$


и, по существу, совпадает с уже решенной задачей (3.6). Сопоставляя (3.6) и (4.17), заключаем, что собственные числа задачи (4.17) записываются в виде (4.15). Лемма доказана.

Отметим, что в леммах 4 и 5 мы не налагали никаких дополнительных ограничений на число $p>0$.

Определим оператор $R=I-\lambda_{0} A_{\mathrm{B}} G^{\prime \prime}\left(x_{0}\right): L^{2} \rightarrow L^{2}$; этот оператор в рассматриваемой нами ситуации, очевидно, играет роль оператора $R_{1}$ из условия $\mathbf{C} 4$.

4.4. Вычисление ядра оператора $R$. При проверке выполнения условия С4 важное значение имеет следующее утверждение.

ЛЕмма 6. 1. Пусть фиксировано число $p>0$ такое, что выполнено условие невырожденности (1.4). Тогда оператор $R$ невырожден, m.e. Ker $R=\{0\}$.

2. При $p=2$ ядро оператора $R$ представляет собой одномерное подпространство в $L^{2}$, натянутое на вектор $\varphi_{0}$ из бормуль (3.5), при этом $\varphi_{0} \equiv x_{0}$.

3. Пусть число $p>2$ таково, что не выполнено (1.4), т.е. $p=2+\frac{4 \pi^{2} n^{2}}{\beta^{2} \omega^{2}}$ при некотором $n \in \mathbb{N}$. Тогда ядро оператора $R$ представляет собой двумерное подпространство в $L^{2}$, натянутое на векторы $\varphi_{n} u \varphi_{-n}$, заданные в формуле (3.5).

ДокАЗАтЕльство. Зафиксируем число $p>0$ и для $y \in L^{2}$ решим уравнение $[I-$ $\left.\lambda_{0} A_{\mathrm{B}} G^{\prime \prime}\left(x_{0}\right)\right] y=0$. Согласно формулам (4.12) и (4.14) это уравнение равносильно уравнению

$$
m \omega^{2}(p-1) A_{\mathrm{B}} y=y, \quad y \in L^{2} .
$$

При $p=1$ данное уравнение, очевидно, имеет единственное решение $y=0$. Пусть $p \neq 1$. Сопоставляя задачу (3.6) и уравнение (4.18), заключаем, что ядро оператора $R$ является вырожденным тогда и только тогда, когда число $p>0$ удовлетворяет равенству $\left[m \omega^{2}(p-1)\right]^{-1}=\nu_{n}$ при некотором $n=0, \pm 1, \pm 2, \ldots$, где $\nu_{n}$ определено в формуле (3.4). Отсюда и из леммы 2 вытекают утверждения леммы.

4.5. Вычисление $\operatorname{det} R$. Нам понадобится следующее утверждение.

ЛЕмма 7. 1. Пусть фиксированы комплексные числа а $u b, b \neq \pm 1, \pm 2, \ldots$. Тогда

$$
\prod_{k=1}^{\infty}\left(1+\frac{a}{k+b}\right)\left(1-\frac{a}{k-b}\right)=\frac{b \pi}{\sin \pi b}\left\{\begin{array}{lll}
\frac{\sin [(a+b) \pi]}{(a+b) \pi}, & \text { eсли } & a+b \neq 0 \\
1, & \text { если } & a+b=0 .
\end{array}\right.
$$

2. Пусть фиксированы действительные числа $c>0$ и

$$
\prod_{k=1}^{\infty}\left(1-\frac{d}{k^{2}+c^{2}}\right)=\frac{c}{\operatorname{sh} \pi c}\left\{\begin{array}{lll}
\frac{\sin \left(\pi \sqrt{d-c^{2}}\right)}{\sqrt{d-c^{2}}}, & \text { если } & d \neq c^{2} \\
\pi, & \text { если } & d=c^{2}
\end{array}\right.
$$

ДокАЗАТЕльство. Формула (4.19) приведена в справочнике [38] (см. п. 6.2.1, № 12); опечатка, допущенная в [38], нами исправлена. Отметим, что соотношение при $a+b=0$ в формуле (4.19) получается в результате предельного перехода 
$a+b \rightarrow 0$. Положив в формуле (4.19) $a=-i c+\sqrt{d-c^{2}}, b=i c$, где $i=\sqrt{-1}$, получаем после несложных вычислений соотношения (4.20). Отметим, что формула (4.20) при $d<c^{2}$ получена иным способом в статье [2].

ЛЕмма 8. 1. Пусть биксировано число $0<p<2$. Тогда справедливо равенство

$$
\operatorname{det} R \equiv \operatorname{det}\left[I-\lambda_{0} A_{\mathrm{B}} G^{\prime \prime}\left(x_{0}\right)\right]=\frac{\operatorname{sh}^{2}\left(\frac{\beta \omega}{2} \sqrt{2-p}\right)}{\operatorname{sh}^{2} \frac{\beta \omega}{2}} .
$$

2. При $p=2$ для чисел $\gamma_{k}$, заданных в (4.15), и $\lambda_{0}=m \omega^{2} / 2$ выполнено равенство

$$
\operatorname{det} R_{0}:=\prod_{\substack{-\infty<<<+\infty \\ k \neq 0}}\left(1-\lambda_{0} \gamma_{k}\right)=\frac{\beta^{2} \omega^{2}}{4 \operatorname{sh}^{2} \frac{\beta \omega}{2}}
$$

3. Пусть фиксировано число $p>2$. Тогда справедливо равенство

$$
\operatorname{det} R=-\frac{\sin ^{2}\left(\frac{\beta \omega}{2} \sqrt{p-2}\right)}{\operatorname{sh}^{2} \frac{\beta \omega}{2}} .
$$

ДокАЗАТЕЛЬСтво. Для $p>0, p \neq 2$, согласно формулам (4.12) и (4.15) получаем соотношения

$$
\operatorname{det} R \equiv \prod_{k=-\infty}^{\infty}\left(1-\lambda_{0} \gamma_{k}\right)=(2-p)\left[\prod_{k=1}^{\infty}\left(1-\frac{(p-1) \beta^{2} \omega^{2}}{\beta^{2} \omega^{2}+4 \pi^{2} k^{2}}\right)\right]^{2}
$$

Если $p=2$, то

$$
\operatorname{det} R_{0}=\left[\prod_{k=1}^{\infty}\left(1-\frac{\beta^{2} \omega^{2}}{\beta^{2} \omega^{2}+4 \pi^{2} k^{2}}\right)\right]^{2}
$$

$\mathrm{K}$ произведениям из последних двух равенств примени́м пункт 2 леммы 7 с параметрами $c=\beta \omega / 2 \pi, d=(p-1) \beta^{2} \omega^{2} / 4 \pi^{2}$. Для этих чисел справедливо равенство $d-c^{2}=(p-2) \beta^{2} \omega^{2} / 4 \pi^{2}$. Используя последнее равенство и выполняя простые вычисления, убеждаемся, что из формул (4.20) вытекают соотношения (4.21)-(4.23). Лемма доказана.

Отметим, что детерминант (4.25) равен нулю для чисел $p>2$ вида (1.9).

\section{6. Вычисление $\left\langle R^{-1} x_{0}, A_{\mathrm{B}}^{-1} x_{0}\right\rangle$ в невырожденном случае.}

ЛЕмма 9. Пусть фиксировано число $р>0$ такое, что выполнено условие невърожденности (1.4). Тогда уравнение $\left[I-\lambda_{0} A_{\mathrm{B}} G^{\prime \prime}\left(x_{0}\right)\right] y=x_{0}, y \in L^{2}$, имеет единственное решение

$$
y_{0}(t) \equiv R^{-1} x_{0}=\frac{x_{0}(t)}{2-p}, \quad t \in[0,1] .
$$

Справедливо равенство

$$
\left\langle R^{-1} x_{0}, A_{\mathrm{B}}^{-1} x_{0}\right\rangle=\frac{m \omega^{2} \beta^{(p-2) / p}}{2-p} .
$$


ДокАЗАТЕЛЬство. Согласно п. 1 леммы 6 для рассматриваемых значений $p$ на всем $L^{2}$ однозначно определен оператор $R^{-1}$. Используя равенство $x_{0}=\lambda_{0} A_{\mathrm{B}} G^{\prime}\left(x_{0}\right)$ и формулы (4.2), (4.5), имеем $x_{0}=\lambda_{0} p \beta^{(2-p) / p} A_{\mathrm{B}} x_{0}$. В силу формулы (4.14) справедливо равенство $A_{\mathrm{B}} G^{\prime \prime}\left(x_{0}\right) x_{0}=p(p-1) \beta^{(2-p) / p} A_{\mathrm{B}} x_{0}$. Из последних двух соотношений следует, что $\lambda_{0} A_{\mathrm{B}} G^{\prime \prime}\left(x_{0}\right) x_{0}=(p-1) x_{0}$. Отсюда получаем формулу $\left[I-\lambda_{0} A_{\mathrm{B}} G^{\prime \prime}\left(x_{0}\right)\right] x_{0}=(2-p) x_{0}$, из которой вытекает (4.26). Используя соотношения (4.13) и (4.26), получаем (4.27). Лемма доказана.

4.7. Доказательство п. 1 теоремы 3. Рассмотрим сначала случай $2>p \geqslant 1$.

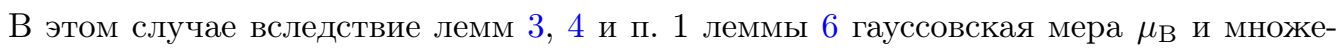
ство (4.4) удовлетворяют условиям C1-C4 с $H=L^{2}, m=2, x^{1}=x_{0}$ и $x^{2}=-x_{0}$. Таким образом, применима теорема 6 , и мы можем использовать соотношение (2.5). Несложно увидеть, что правые части формулы (2.5) для обеих экстремальных функций совпадают, поэтому достаточно провести вычисления только для точки $x_{0}$. Подставляя выражения из формул $(4.13),(4.21)$ и (4.27) в соотношение (2.5), мы получаем формулы (1.12) и (1.13). Таким образом, п. 1 теоремы 3 доказан для значений $2>p \geqslant 1$.

Пусть теперь $1>p>0$. В этом случае для меры $\mu_{\mathrm{B}}$ и множества (4.4) выполнены условия $\widetilde{\mathbf{C 1}}, \mathbf{C 2 -} \mathbf{C 4}$ также с $H=L^{2}, m=2, x^{1}=x_{0}$ и $x^{2}=-x_{0}$. Применяя теорему $6^{\prime}$ и формулы (4.13), (4.21), (4.27), мы снова получаем соотношения (1.12) и (1.13). Пункт 1 теоремы 3 доказан полностью.

4.8. Доказательство п. 2 теоремы 3. Пусть $p=2$. В этом случае вследствие

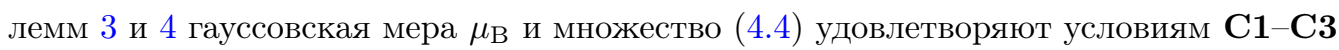
с $H=L^{2}, m=2, x^{1}=x_{0}$ и $x^{2}=-x_{0}$. Покажем, что применима теорема 7 . Рассмотрим случай экстремальной точки $x_{0}$; рассуждения и вычисления в случае второй точки $-x_{0}$ такие же. Согласно п. 2 леммы 6 ядро оператора $R=I-\lambda_{0} A_{\mathrm{B}} G^{\prime \prime}\left(x_{0}\right)$ представляет собой одномерное подпространство в $L^{2}$, натянутое на вектор $x_{0}$. В силу формулы (4.13) и условия нормированности функции $x_{0}$ справедливы равенства

$$
\left\langle x_{0}, x_{0}\right\rangle=1, \quad\left\langle x_{0}, A_{\mathrm{B}}^{-1} x_{0}\right\rangle=m \omega^{2} .
$$

Таким образом, выполнено условие $\widetilde{\mathbf{C 4}}$ и применима теорема 7 с $H=L^{2}, m=2$, $x^{1}=x_{0}, x^{2}=-x_{0}, e_{1}=e_{2}=x_{0}$. Используем соотношения (2.6) и (2.7). Здесь также правые части формулы (2.6) для обеих экстремальных функций совпадают. Несложно убедиться, что для определителей из формулы (2.7) выполнены равенства $\operatorname{det} R_{10}=\operatorname{det} R_{20}=\operatorname{det} R_{0}$, где $\operatorname{det} R_{0}$ задан в соотношении (4.22). Подставляя выражения из формул (4.13), (4.22) и (4.28) в соотношение (2.6), мы получаем (1.14). Пункт 2 теоремы 3 доказан.

4.9. Доказательство п. 3 теоремы 3. Пусть фиксировано число $p>2$ такое, что выполнено (1.4). В этом случае вследствие лемм 3, 4 и п. 1 леммы 6 гауссовская мера $\mu_{\mathrm{B}}$ и множество (4.4) удовлетворяют условиям C1-C4 с $H=L^{2}, m=2, x^{1}=x_{0}$ и $x^{2}=-x_{0}$. Таким образом, применима теорема 6 , и мы можем снова использовать соотношение (2.5). Здесь также правые части формулы (2.5) для обеих экстремальных функций совпадают. Подставляя выражения из формул (4.13), (4.23) и (4.27) 
в соотношение (2.5), мы получаем формулы (1.15) и (1.16). Теорема 3 полностью доказана.

Отметим, что п. 2 теоремы 3 можно доказать также при помощи известного результата Золотарева, см. [39] или теорему 3.8 (п. 2) в [18].

\section{5. ДОКАЗАТЕЛЬСТВО ТЕОРЕМЫ 4 И ФОРМУЛ (1.18), (1.19)}

Доказательство теоремы 4 проводится по той же схеме, что и доказательство теоремы 3 на основе теоремы $6^{\prime}$. При этом существенно используются утверждения, полученные в разделе 4. Приведем основные моменты. В качестве гильбертова пространства $H$, участвующего в формулировке теоремы $6^{\prime}$, возьмем опять пространство $L^{2}$. В качестве функционала $Q$ возьмем функционал

$$
G_{1}(x):=\int_{0}^{\beta} x^{p}(t) d t-1, \quad x \in L^{2} .
$$

Этот функционал не является выпуклым. Отметим, что в случае такого функционала экстремальная задача из условия С2 имеет единственное решение - функцию $x_{0}(t)$, заданную в формуле (4.5). В этом можно убедиться, обратившись к доказательству леммы 4. Для функционалов (4.1) и (5.1) справедливы равенства $G\left(x_{0}\right)=G_{1}\left(x_{0}\right), G^{\prime}\left(x_{0}\right)=G_{1}^{\prime}\left(x_{0}\right), G^{\prime \prime}\left(x_{0}\right)=G_{1}^{\prime \prime}\left(x_{0}\right)$. Поэтому утверждения лемм $5-9$ остаются справедливыми и для оператора $R_{1}:=I-\lambda_{0} A_{\mathrm{B}} G_{1}^{\prime \prime}\left(x_{0}\right) \equiv R$.

Далее, предполагая выполненными условия теоремы 4 , применяем теорему $6^{\prime}$ с $H=L^{2}, m=1, x^{1}=x_{0}$. Сравнивая полученные в результате соотношения с формулами (1.12), (1.15), приходим к формуле (1.17). Теорема 4 доказана.

Доказательство формул (1.18) и (1.19) проводится при помощи леммы 4 и принципа больших уклонений для гауссовских мер (см., например, [8], [11], [18]).

\section{6. ДОКАЗАТЕЛЬСТВО ТЕОРЕМ 1 И 2}

Сначала мы изложим доказательство теоремы 1 , которое проведем при помощи теоремы 5 .

6.1. Выбор банахова функционального пространства. Для $p>2$ не удается ограничиться только гильбертовым пространством $L^{2}[0, \beta]$ и нужно привлекать пространства $L^{p}[0, \beta]$. Зафиксируем числа $p>0$ и $0<\alpha<2$. Положим

$$
\tilde{p}:=\left\{\begin{array}{lll}
p, & \text { если } & p>2, \\
2, & \text { если } 0<p \leqslant 2 .
\end{array}\right.
$$

Возьмем в качестве банахова пространства $E$ из теоремы 5 банахово пространство $L^{\tilde{p}} \equiv L^{\tilde{p}}[0, \beta]$ функций, $\tilde{p}$-я степень которых интегрируема на отрезке $[0, \beta]$. Норма в $L^{\tilde{p}}$ обозначается через $\|\cdot\|_{\tilde{p}}$ и определена в (1.8). Очевидно, $\tilde{p} \geqslant 2$. Пусть $2 \geqslant \tilde{q}>1$ обозначает число, сопряженное к $\tilde{p}$, т.е. $1 / \tilde{p}+1 / \tilde{q}=1$. Напомним, что банахово пространство $L^{\tilde{q}}$ является сопряженным к пространству $L^{\tilde{p}}$.

В данном разделе мы будем полагать, что гауссовская мера Боголюбова $\mu_{\mathrm{B}}$ задана в пространстве $L^{\tilde{p}}[0, \beta]$, имея в виду, что эта мера сосредоточена на $C^{0}[0, \beta]$. 
Известно, что в этом случае мера $\mu_{\mathrm{B}}$ имеет нулевое среднее и ковариационный оператор $A_{\mathrm{B}}: L^{\tilde{q}} \rightarrow L^{\tilde{p}}$ интегрального типа, который определяется по формуле (3.1) для $x \in L^{\tilde{q}}$. Здесь также справедлива лемма 1. Отметим, что теперь оператор $A_{\mathrm{B}}$ зависит от $\tilde{p}$, в частности $\operatorname{Dom} A_{\mathrm{B}}=L^{\tilde{q}}$, но указывать эту зависимость мы не будем. Будем теперь обозначать через $\langle\cdot, \cdot\rangle$ каноническую билинейную форму между пространствами $L^{\tilde{p}}$ и $L^{\tilde{q}}$, которая, как известно, имеет интегральный вид: $\langle h, g\rangle=\int_{0}^{1} h(t) g(t) d t$ для $h \in L^{\tilde{p}}, g \in L^{\tilde{q}}$.

Нам понадобится следующее полезное неравенство для функционала (1.8).

Лемма 10. Пусть фиксированъ числа $0<s<r<\infty$. Если $\|x\|_{r}<\infty$, то справедливо неравенство $\|x\|_{s} \leqslant \beta^{(r-s) / r s}\|x\|_{r}$.

ДокАЗАТЕЛьСтво леммы имеется, например, в книгах [30] и [34].

6.2. Выбор основного функционала. Мы докажем теорему 1 , применяя теорему 5 , в которой будем полагать $E=L^{\tilde{p}}, P_{A}=\mu_{\mathrm{B}}, A=A_{\mathrm{B}}$ и $f \equiv 1$.

Возьмем в качестве функционала $F$, участвующего в формулировке теоремы 5 , следующий функционал:

$$
\Psi(x):=-\|x\|_{p}^{\alpha} \equiv-\left(\int_{0}^{\beta}|x(t)|^{p} d t\right)^{\alpha / p}, \quad x \in L^{\tilde{p}} .
$$

Наша цель - применить теорему 5 к функционалу (6.2) и гауссовской мере Боголюбова $\mu_{\mathrm{B}}$. Положим

$$
v=u^{1 /(2-\alpha)}
$$

Тогда $u=v^{2-\alpha}$, кроме того, $v \rightarrow \infty$ при $u \rightarrow \infty$. Функционал (6.2) обладает свойством однородности порядка $\alpha: \Psi(r x)=r^{\alpha} \Psi(x), r>0$. Используя это свойство и равенство (6.3), запишем среднее из формулы (1.3) в виде модельного интеграла Лапласа:

$$
\mathbf{E} \exp \left\{u\left(\int_{0}^{\beta}|\xi(t)|^{p} d t\right)^{\alpha / p}\right\}=\mathbf{E} e^{-u \Psi(\xi)}=\int_{L^{\tilde{p}}} e^{-v^{2} \Psi(x)} d \mu_{\mathrm{B}}(v x) .
$$

Функционал $-\Psi(x)$ представляет собой степень интегрального функционала. Как известно, интегральные функционалы дифференцируемы по Фреше (см. [25], [26], [34]). Напомним, что множество $\mathcal{X}$ было определено перед леммой 3.

ЛЕмма 11. Функиионал $\Psi$ трижды дифферениируем по Фреше в каждой точке $x \in \mathcal{X}$, при этом первые две производные имеют следующий вид:

$$
\begin{gathered}
\Psi^{\prime}(x)=-\alpha\left(\int_{0}^{\beta}|x(s)|^{p} d s\right)^{(\alpha-p) / p}|x(t)|^{p-1} \operatorname{sign} x(t), \\
\Psi^{\prime \prime}(x)[h, g]=\alpha(1-p)\|x\|_{p}^{\alpha-p}\left\langle h,|x(t)|^{p-2} g\right\rangle+ \\
+\alpha(p-\alpha)\|x\|_{p}^{\alpha-2 p}\left\langle h,|x(t)|^{p-1} \operatorname{sign} x(t)\right\rangle \times \\
\quad \times\left\langle g,|x(t)|^{p-1} \operatorname{sign} x(t)\right\rangle, \quad h, g \in L^{\tilde{p}} .
\end{gathered}
$$


При $x \in \mathcal{X}$ определен линейный оператор $\Psi^{\prime \prime}(x): L^{\tilde{p}} \rightarrow L^{\tilde{q}}$, действующий по правилу

$$
\begin{aligned}
\Psi^{\prime \prime}(x) g= & \alpha(1-p)\|x\|_{p}^{\alpha-p}|x(t)|^{p-2} g+ \\
& +\alpha(p-\alpha)\|x\|_{p}^{\alpha-2 p}|x(t)|^{p-1} \operatorname{sign} x(t)\left\langle g,|x(t)|^{p-1} \operatorname{sign} x(t)\right\rangle, \quad g \in L^{\tilde{p}}
\end{aligned}
$$

ДокАзАТЕльство. Утверждение леммы получаем, применяя теорему о суперпозиции дифференцируемых отображений [26] и известные формулы для первых двух производных функционала $G_{0}(x)=\int_{0}^{\beta}|x(t)|^{p} d t$, см. лемму 3 и [26], [34]. При этом формула (6.6), очевидно, вытекает из равенств (6.5).

ЛЕмма 12. Для функиионала $\Psi$ выполнено условие D1.

ДоКАЗАТЕЛЬСтво леммы проводится при помощи леммы 10.

6.3. Решение экстремальной задачи. Решим экстремальную задачу из условия D2 для функционала (6.2) и ковариационного оператора $A_{\mathrm{B}}$. Введем следующие положительные величины:

$$
\sigma^{2}=\sigma^{2}(p)=\frac{\beta^{(2-p) / p}}{m \omega^{2}}, \quad b_{0}=b_{0}(p, \alpha)=\left(\alpha \sigma^{2}\right)^{1 /(2-\alpha)} .
$$

Определим постоянную строго положительную функцию

$$
x_{1}(t):=b_{0} x_{0}(t)=\left(\frac{\alpha}{m \omega^{2}}\right)^{1 /(2-\alpha)} \beta^{(\alpha-p) /(p(2-\alpha))}, \quad t \in[0, \beta],
$$

где функция $x_{0}(t)$ задана в формуле (4.5). Таким образом, $x_{1} \in \mathcal{X}$. Несложно видеть, что $\left\|x_{1}\right\|_{p}=b_{0}$.

Лемма 13. Пусть фиксированы $p>0 u 0<\alpha<2$. Тогда функционал $\Lambda(x):=$ $\Psi(x)+\left\langle x, A_{\mathrm{B}}^{-1} x\right\rangle / 2$ достигает на Dom $A_{\mathrm{B}}^{-1}$ своего минимума, равного $-q_{0}$, в двух точках $x_{1}(t)$ u $-x_{1}(t)$, где число $q_{0}$ определено в бормуле (1.2). При этом $\inf _{x \in H_{A_{\mathrm{B}}}}\left[\Psi(x)+\|x\|_{A_{\mathrm{B}}}^{2} / 2\right]$ также достигается только в двух точках $x_{1}(t) u-x_{1}(t)$.

ДокАзАТЕльство. Переходя к условной экстремальной задаче, убеждаемся, что справедливы следующие равенства:

$$
\begin{aligned}
\inf _{x \in \operatorname{Dom} A_{\mathrm{B}}^{-1}}\left[\frac{1}{2}\left\langle x, A_{\mathrm{B}}^{-1} x\right\rangle-\|x\|_{p}^{\alpha}\right] & =\inf _{b>0} \inf _{\substack{x \in \operatorname{Dom}_{\mathrm{B}}^{-1},\|x\|_{p}=b}}\left[\frac{1}{2}\left\langle x, A_{\mathrm{B}}^{-1} x\right\rangle-\|x\|_{p}^{\alpha}\right]= \\
& =\inf _{b>0} \inf _{\substack{x \in \operatorname{Dom}_{\mathrm{B}}^{-1},\|x\|_{p}=b}}\left[\frac{1}{2}\left\langle x, A_{\mathrm{B}}^{-1} x\right\rangle-b^{\alpha}\right]= \\
& =\inf _{b>0}\left(\frac{b^{2}}{2 \sigma^{2}}-b^{\alpha}\right)=\frac{b_{0}^{2}}{2 \sigma^{2}}-b_{0}^{\alpha}=-q_{0} .
\end{aligned}
$$

Несложно проверить, что минимум в (6.9) достигается только в двух точках $x_{1}(t)$ и $-x_{1}(t)$. 
6.4. Исследование спектра интегрального оператора $A_{\mathrm{B}} \Psi^{\prime \prime}\left(x_{1}\right)$. При

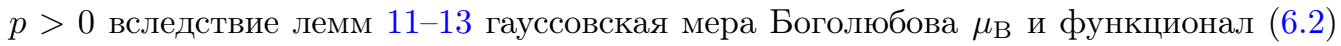
удовлетворяют условиям D1-D3 с $E=L^{\tilde{p}}, f \equiv 1, F=\Psi, m=2, x^{1}=x_{1}>0$ и $x^{2}=-x_{1}$.

Рассмотрим условие D4. Согласно формуле (6.6) имеет место равенство $\Psi^{\prime \prime}\left(x_{1}\right)=$ $\Psi^{\prime \prime}\left(-x_{1}\right)$, поэтому достаточно исследовать спектр интегрального оператора $A_{\mathrm{B}} \Psi^{\prime \prime}\left(x_{1}\right): L^{\tilde{p}} \rightarrow L^{\tilde{p}}$. Этот оператор является ядерным как композиция ядерного оператора $A_{\mathrm{B}}$ и ограниченного оператора $\Psi^{\prime \prime}\left(x_{1}\right)$ [37]. Учитывая равенство $\left\|x_{1}\right\|_{p}=$ $b_{0}$ и используя формулы (6.6)-(6.8), для $t \in[0, \beta], z \in L^{\tilde{p}}$ получаем соотношение

$$
\left[\Psi^{\prime \prime}\left(x_{1}\right) z\right](t)=(1-p) m \omega^{2} z(t)+(p-\alpha) \frac{m \omega^{2}}{\beta} \int_{0}^{\beta} z(s) d s .
$$

Лемма 14. Пусть фиксированы $p>0 u 0<\alpha<2$. Тогда собственные значения $\left\{\gamma_{n}\right\}_{n=-\infty}^{\infty}$ ядерного интегрального оператора $A_{\mathrm{B}} \Psi^{\prime \prime}\left(x_{1}\right)$ имеют следующий вид:

$$
\gamma_{0}=1-\alpha, \quad \gamma_{n}=\frac{(1-p) \omega^{2}}{\omega^{2}+\left(2 \pi \beta^{-1} n\right)^{2}}, \quad n= \pm 1, \pm 2, \ldots
$$

ДокАЗАТЕЛЬСтво проводится аналогично доказательству леммы 5 при помощи леммы 1 и формулы (6.10). Отметим, что в леммах 11-14 мы не налагали никаких дополнительных ограничений на число $p>0$.

Определим оператор $R=I+A_{\mathrm{B}} \Psi^{\prime \prime}\left(x_{1}\right): L^{\tilde{p}} \rightarrow L^{\tilde{p}}$; этот оператор в рассматриваемой нами задаче играет роль оператора $R_{1}$ из условия $\mathbf{D} 4$.

\section{5. Вычисление определителя оператора $R$.}

ЛЕмма 15. Пусть задано число $0<\alpha<2$. Тогда справедливы следующие утверждения.

1. Предположим, что фиксировано число $0<p<2$. Тогда

$$
\operatorname{det} R \equiv \prod_{n=-\infty}^{\infty}\left(1+\gamma_{n}\right)=\frac{(2-\alpha) \operatorname{sh}^{2}\left(\frac{\beta \omega}{2} \sqrt{2-p}\right)}{(2-p) \operatorname{sh}^{2} \frac{\beta \omega}{2}}
$$

2. При $р=2$ выполнено равенство

$$
\operatorname{det} R=\frac{(2-\alpha) \beta^{2} \omega^{2}}{4 \operatorname{sh}^{2} \frac{\beta \omega}{2}}
$$

3. Пусть фиксировано число $p>2$. Тогда

$$
\operatorname{det} R=\frac{(2-\alpha) \sin ^{2}\left(\frac{\beta \omega}{2} \sqrt{p-2}\right)}{(p-2) \operatorname{sh}^{2} \frac{\beta \omega}{2}} .
$$

4. Для оператора $A_{\mathrm{B}} \Psi^{\prime \prime}\left(x_{1}\right)$ выполнено условие D4 при всех $p>0$, удовлетворяющих условию невырожденности (1.4).

ДокАзАТЕЛЬство первых трех утверждений проводится аналогично доказательству леммы 8 при помощи леммы 14 и п. 2 леммы 7. Утверждение 4 леммы вытекает из первых трех и того факта, что детерминант (6.11) равен нулю для чисел $p>2$ таких, что $p=2+\frac{4 \pi^{2} n^{2}}{\beta^{2} \omega^{2}}$ при некотором $n \in \mathbb{N}$. 
6.6. Доказательство теоремы 1. Пусть фиксированы число $0<\alpha<2$ и число $p>0$ такое, что выполнено условие невырожденности (1.4). Тогда вследствие лемм 11-15 гауссовская мера Боголюбова $\mu_{\mathrm{B}}$ и функционал (6.2) удовлетворяют условиям D1-D4 с $E=L^{\tilde{p}}, f \equiv 1, F=\Psi, m=2, x^{1}=x_{1}$ и $x^{2}=-x_{1}$. Таким образом, к интегралу (6.4) применима теорема 5, и мы можем использовать соотношение (2.1). Несложно видеть, что правые части формулы (2.1) для обеих экстремальных функций совпадают, поэтому достаточно провести вычисления только для точки $x_{1}$. Используя леммы 13,15 и формулы $(2.1),(6.3)$, мы получаем утверждения 1-3 теоремы 1 . Теорема 1 доказана.

6.7. Доказательство теоремы 2 и формул (1.10), (1.11). Доказательство теоремы 2 проводится на основе теоремы 5 по той же схеме, что и доказательство теоремы 1. При этом существенным образом используются утверждения, полученные в разделе 6 . Отметим, что здесь в качестве функционала $F$ берется функционал $\Psi_{1}(x):=-\left(\int_{0}^{\beta} x^{p}(t) d t\right)^{\alpha / p}, x \in L^{\tilde{p}}$. При этом соответствующая экстремальная задача из условия $\mathbf{D} 2$ имеет единственное решение - функцию $x_{1}(t)$, заданную в (6.8).

Доказательство формул (1.10), (1.11) проводится при помощи леммы 13 и принципа больших уклонений для гауссовских мер в интегральной форме (теоремы Варадана), см. [8], [18].

Благодарности. Работа выполнена при поддержке РФФИ (грант № 04-0100700).

\section{Список литературы}

[1] Д. П. Санкович, ТМФ, 119:2 (1999), 345-352.

[2] Д. П. Санкович, ТМФ, 126:1 (2001), 149-163.

[3] Д. П. Санкович, ТМФ, 127:1 (2001), 125-142.

[4] Н.Н. Боголюбов, ДАН СССР, 99 (1954), 225-226; А. В. Свидзинский, ЖЭТФ, 31 (1956), 324; Н. Н. Боголюбов, Н. Н. Боголюбов (мл.), Аспекты теории полярона, Сообщения ОИЯИ, P17-81-65, ОИЯИ, Дубна, 1981; G. W. Johnson, M. L. Lapidus, The Feynman Integral and Feynman's Operational Calculus, Clarendon Press, Oxford, 2000.

[5] Г. Крамер, М. Лидбеттер, Стационарные случайные процессы, Мир, М., 1969.

[6] Yu. K. Belayev, "Continuity and Hölder's conditions for sample functions of stationary Gaussian processes", Proc. 4th Berkeley Symp. Math. Stat. Probab., V. II, Univ. California Press, Berkeley, 1961, 23-33.

[7] И. И. Гихман, А. В. Скороход, Теория случайных прощессов, Т. 1, Наука, М., 1971.

[8] М. А. Лифшиц, Гауссовские случайные функиии, ТВиМС, Киев, 1995.

[9] В. И. Питербарг, Асимптотические методы в теории гауссовских случайных процессов и полей, Изд-во МГУ, М., 1988.

[10] Х.-С. Го, Гауссовские меры в банаховых пространствах, Мир, М., 1979.

[11] В. И. Богачев, Гауссовские меры, Наука, М., 1997.

[12] Н. Н. Вахания, В.И. Тариеладзе, С. А. Чобанян, Вероятностные распределения в банаховых пространствах, Наука, М., 1985.

[13] А.Д. Егоров, Л.А. Янович, ДАН БССР, 14 (1970), 873-876; П. И. Соболевский, Л. А. Янович, ДАН БССР, 18 (1974), 965-968.

[14] F.W. Wiegel, Phys. Rep., 16:2 (1975), 57-114; Л.А. Пастур, ТМФ, 32:1 (1977), 88-95; L.S. Schulman, Techniques and Applications of Path Integration, Wiley, New York, 1981; M. D. Donsker, S. R. S. Varadhan, Comm. Pure Appl. Math., 36:4 (1983), 505-528; 
В. П. Маслов, Тр. МИАН, 163 (1984), 150-180; М. I. Freidlin, Functional Integration and Partial Differential Equations, Ann. of Math. Stud., 109, Princeton Univ. Press, Princeton, 1985; Дж. Льюис, К. Фистер, УМН, 50:2(302) (1995), 47-88.

[15] B. Simon, Functional Integration and Quantum Physics, Pure Appl. Math., 86, Academic Press, New York, 1979.

[16] R.S. Ellis, J.S. Rosen, Trans. Amer. Math. Soc., 273:2 (1982), 447-481.

[17] R. S. Ellis, J. S. Rosen, Ann. Probab., 10:1 (1982), 47-66; Comm. Math. Phys., 82:2 (1981), $153-181$.

[18] В. И. Питербарг, В. Р. Фаталов, УМН, 50:6 (1995), 57-150.

[19] В.Р. Фаталов, ТВП, 41:3 (1996), 682-689; 51:3 (2006), 634-636.

[20] В.Р. Фаталов, “Точные асимптотики винеровских интегралов типа Лапласа для $L^{p}$-функционалов, $p>0$ ", Изв. РАН, Сер. матем. (в печати).

[21] В.Р. Фаталов, Матем. сб., 196:4 (2005), 135-160; Пробл. передачи информ., 42:1 (2006), 52-71.

[22] Т. Хида, Броуновское движение, Наука, М., 1987.

[23] Н. Икэда, С. Ватанабэ, Стохастические дифференииальные уравнения и диффузионные прочессы, Наука, М., 1986.

[24] В. Р. Фаталов, Изв. НАН Армении. Математика, 27:5 (1992), 43-61.

[25] М. М. Вайнберг, Вариационный метод и метод монотонных операторов, Наука, М., 1972 .

[26] В. М. Алексеев, В. М. Тихомиров, С. В. Фомин, Оптимальное управление, Наука, М., 1979.

[27] И. Ц. Гохберг, М. Г. Крейн, Введение в теорию линейных несамосопряженных операторов, Наука, М., 1965.

[28] М. Рид, Б. Саймон, Методы современной математической физики, Т. 4, Мир, М., 1982.

[29] В.А. Садовничий, Теория операторов, Изд-во МГУ, М., 1979.

[30] Л. В. Канторович, Г. П. Акилов, Функииональный анализ, Наука, М., 1977.

[31] S. Chevet, "Gaussian Measures and Large Deviations", Probability in Banach spaces IV, Lecture Notes in Math., 990, eds. A. Beck, K. Jacobs, Springer, Berlin, 1983, 30-46.

[32] А.Д. Полянин, А.В. Манжиров, Справочник по интегралъным уравнениям: Точные решения, Факториал, М., 1998.

[33] Э. Камке, Справочник по обыкновенным дифференциальным уравнениям, Наука, М., 1961.

[34] М. А. Красносельский, П. П. Забрейко, Е. И. Пустыльник, П. Е. Соболевский, Интегральные операторы в пространствах суммируемых функций, Наука, М., 1966.

[35] С. Г. Крейн (ред.), Функциональный анализ, Наука, М., 1972.

[36] Ф. Рисс, Б. Сёкефальви-Надь, Лекиии по функииональному анализу, Мир, М., 1979.

[37] А. Пич, Операторные идеалы, Мир, М., 1982.

[38] А.П. Прудников, Ю.А. Брычков, О. И. Маричев, Интегралы и ряды. Элементарные функиии, Наука, М., 1981.

[39] В. М. Золотарев, ТВП, 6:2 (1961), 219-222. 